\title{
Exploring Computational Scheme of Complex Problem Solving Based on Meta-Synthesis Approach
}

\author{
Yijun Liu ${ }^{1}$, Wenyuan $\mathrm{Niu}^{1}$, and Jifa $\mathrm{Gu}^{2}$ \\ ${ }^{1}$ Institute of Policy and Management, Chinese Academy of Sciences, Beijing 100080 P.R. China \\ yijunliuacasipm.ac.cn \\ ${ }^{2}$ Institute of Systems Science, Academy of Mathematics and Systems Science \\ Chinese Academy of Sciences, Beijing 100080 P.R. China
}

\begin{abstract}
There are many approaches to complex problem solving, depending on the nature of the problem and the people involved in the problem. In this paper, a computational scheme based on meta-synthesis approach is applied to a social problem on exploring some issues about taxis drivers. Firstly, group argumentation environment (GAE) as a computerized tool is used to facilitate expert thinking process (meeting) about the concerned topics and form scenario or hypotheses based on qualitative meta-synthesis approach. Next, six strategies of quantitative meta-synthetic system modeling are introduced and one of that is used to construct a multi-agent model which aims to analyze the relations between taxis drivers' income and their behavior response intensity. Finally, some concluding remarks and future works are given.
\end{abstract}

Keywords: meta-synthesis, group argumentation, multi-agent system.

\section{Introduction}

Meta-synthesis approach (MSA) is proposed to tackle with complex, open and giant systems by Chinese scientists Qian, X.S. and his colleagues around the start of 1990s, which expects "to unite organically the expert group, data, all sorts of information, and the computer technology, and to unite scientific theory of various disciplines and human experience and knowledge" for proposing hypothesis and quantitative validating [1]. The essential idea of MSA can be simplified as "confident hypothesizing, rigorous validating", i.e. quantitative knowledge arises from qualitative understanding, which reflects the process of knowing and doing in epistemology. Later the concept of Hall of Workshop for Meta-Synthetic Engineering (HWMSE) is proposed as MSA practicing platform which is expected to utilize breaking advances in information technologies while the active roles of human beings are greatly emphasized during human-machine collaboration [2,3]. There are three kinds of meta-synthesis, 1) qualitative meta-synthesis; 2) qualitative - quantitative meta-synthesis; 3) meta-synthesis from qualitative hypothesis to quantitative validation. Each kind of meta-synthesis can be supported by various tools or methods.

In this paper, a computational scheme based on meta-synthesis approach is applied to a social problem on exploring some issues about taxis drivers. Firstly, versatile 
computerized aids, such as visualization of expert opinion structure, clustering of contributed opinions for concept formation and automatic affinity diagramming, etc. have been developed and integrated into group argumentation environment (GAE), to facilitate expert thinking process (meeting) about the concerned topics and form scenario or hypotheses based on qualitative meta-synthesis approach. Next, six strategies of quantitative meta-synthetic system modeling are introduced and one of that is used to construct a multi-agent model based on the platform of StarLogo to analyze the relations between taxis drivers' income and their behavior response intensity. Finally, some concluding remarks and future works are given.

\section{Qualitative Analysis for Scenario Forming}

Qualitative meta-synthesis produces scenarios or hypotheses about the complex problems, i.e. to expose some qualitative relations or structures of the concerned problems. A variety of computerized tools, such as group support systems (GSS), creativity support systems (CSS), could be used to support idea generation which is the origin for qualitative meta-synthesis. In recent years, creative group activities have come to the fore with the growing complexity of society. One of aims of divergent thinking is for creative ideas toward unknown issues, hence demand for computerized tools to provide more help during such a thinking process. Here, a computer-based tool, group argumentation environment (GAE), which is an interactive task-oriented cooperation environment to support loosely coupled group's activities, is presented. Absorbing some ideas from AIDE [4], AA1 [5], GAE has two principal modules, i) divergence of visualizing, mainly supporting electronic brainstorming, ii) convergence of computing for the processes and results of group argumentation.

\subsection{Visualized Shared Memory for Group Argumentation}

Brainstorming is a technique, which involves generating a list of ideas in a creative, unstructured manner. The goal of brainstorming is to generate as many ideas as possible in a short period of time. During the brainstorming process, all ideas are recorded, and no idea is disregarded or criticized. After a long list of ideas is generated, one can go back and review the ideas to critique their value or merit. Brainstorming can encourage expert to use their imagination, tap the creativity of the group to capture unique ideas, which may be a little less conventional [4].

Electronic brainstorming is an idea-generating tool in GAE that allows participants to share ideas simultaneously on a specific topic posted to the group. Nowadays, most web-enabled forums can be regarded as simple electronic brainstorming sessions. However, only plain texts are given to all participants. By contrast, in our developed electronic brainstorming module, all collective information, mainly, utterances and keywords by each participant are processed by dual scaling method (a kind of multi-variant statistical method) and results are displayed at a 2-dimension space [6].

Such a visualized map can be understood more easily than plain texts by participants and helpful to expose some relations or structures of the concerning topic, which can also stimulate participants' further emergence of more creative ideas and hold active participation. There are three viewers in GAE. 
- Common Viewer: This discussion space can be regarded as a joint thought space for the participants. All users can participant in argumentation and understand the global structure and relationships by viewing the shared discussion space.

- Personal Viewer: It is an anonymous idea-gathering space in which the relationships between utterances and keywords can be visualized.

- Retrospective Viewer: It applies same mechanism as common viewer and provides participants to "drill down" the discussing process for visualized partial perspectives.

Different from the topological graph, the graph of viewer is an interpretable graph, which reflects the data's nature in the database. But the topological structures have been designed and the forms are structured. In our research, we want to cluster the utterances and keywords of the experts and then externalize the mental process of the human thinking. Here, we think, the interpretable diagram is more suitable to embody the thinking activities than the topological graph [7].

\subsection{Automatic Affinity Diagramming}

The affinity diagramming (sometimes called the KJ diagram after its creator, Kawkita Jiro [8]) was developed to discover meaningful groups of ideas within a raw list. Usually it is used to refine a brainstorming into something that makes sense and can be dealt with more easily. In GAE, an affinity diagramming about the concerned topic discussed in the electronic brainstorming module is automatically produced according to 2-dimension map in the personal viewer. Not only plain text files are saved after a brainstorming session, but a document including a list of affinity sets, which reflects some structure of the concerned topic, is acquired [9].

Automatic affinity diagramming is synchronously working with the participants' discussion. Both visual map and affinity document may be helpful for participants to find some structures of the concerned issues, because visualization can offer users capabilities for self-guided exploration and visual analysis of large amounts of information. Both functions support confident hypothesis formulation during complex problem solving.

\subsection{Concept Formation Based on Centroidal Clustering Algorithm}

Concept formation means automatic summarizing and clustering of experts' utterances and detecting the typical keywords which represent meaningful groups or sub-communities of ideas based on visualized maps. Centroid is the center of each set produced with cluster and given by $C_{m}=\frac{1}{n} \sum_{i=1}^{n} t_{m i}$. Combining K-means clustering method [10], which equation is $m_{i}=\frac{1}{m} \sum_{j=1}^{m} t_{i j}, \mathrm{k}$ centroids, where $\mathrm{k}$ is an assumed number of clusters, can be detected. The closest keyword to the centroid could be regarded as cluster label.

The detailed introduction of other functions of GAE can see the reference $[7,11,12]$. 


\section{Quantitative Computing Based on Meta-Synthetic Modeling}

Given the scenario or hypotheses based on qualitative expert argumentation, modeling is one of the essential and effective computing methods for the complex or un-structured problem. The meta-synthesis approach emphasized during complex system modeling needs to have many kinds of views (perspectives). According to some basic principles of modeling proposed by Ackoff [13], Gu \& Tang summarized the following 6 strategies of modeling which has been used to study the macro-economic problems $[3,14]$.

- Modeling by knowing mechanism: such as input-output models, econometric models for economic forecasting, etc.

- Modeling by analogy: also be called case reasoning or case study. When constructing some new objects or solving the complex problems, we usually consider and absorb the experiments from the existed cases.

- Modeling by rule: typical refers to multi-agent simulation (MAS), which analyzes the group's behavior with that of individuals guided by complex adaptive system theory. At present, many MAS supporting tools, such as SWARM, ASCAPE, REPAST, TNG-lab, AgentSheets and StarLogo, are already here.

- Modeling by data: such as various statistical models, reconstructability analysis (RA) model, etc.

- Modeling by evolution: such as chaos and fractal methods, etc., which helpful to investigate and explore the complexity.

- Modeling by learning: such as models based on data mining and knowledge discovery. Essentially, these models are modeling by data, but modeling by learning emphasizes the hidden structure in the massive data and knowledge, absorbs expert's experience, and improves the models based on data.

Above modeling activities may depend on hypotheses and assumptions given by qualitative meta-synthesis. Next, a whole example for some topics about taxi drivers will be demonstrated to show the computational scheme of social issues solving based on meta-synthesis approach.

\section{A Whole Example of Computational Scheme for Complex Problem Solving}

Taxi has been considered to be one of the most important transportation tools and takes great revenue for taxi companies, but recently, the taxi companies in China have to pay more cost because price of oil has got a continuous rising in global market. A hot discussion on rising in unit price of taxi is accordingly produced. To minimize the effect of cost increasing on public transportation system, governments of many cities, such as Beijing and Shanghai, launched a new solution which controls price of taxi directly depending on the price of oil. At present, the balance between government, taxi companies, and taxi drivers becomes social scale issue which belongs to the complex, open and giant systems. 


\subsection{Qualitative Scenario Forming by View of Group Argumentation}

In this example, the topic for discussion is about the different strategies to deal with the taxi problems under the current situation. All opinions are summarized into 48 utterances contributed by nine persons who participate in the discussion as a community. Fig. 1 shows basic analysis taken in this test. Fig. 1(a) is a whole perspective of all concerned participants' contributions. It shows participants who share more common keywords locate closer in the 2-dimension space. Fig. 1(b) is the opinion structures of partial users as a subset community formed in retrospective viewer. Fig. 1(c) shows the affinity diagramming based on personal viewer, which divides the whole utterance set into several cells according to their locations. It could be seen that the utterances in one cell are related to each other. Automatic affinity diagramming could be regarded as a rough classification about participants' opinions during the brainstorming session. Further processing could be taken to acquire a more reasonable classification. Fig. 1(d) and (e) shows 5 clusters by centroidal clustering algorithm, by which keywords “水流, “访谈”, “信息”, 定量”, and “机理”, are acquired as the label (centroid) of each cluster.

Combining the result of expert argumentation with the expert's background, some methods and strategies to analyze and model the taxi problems are the following:

- Using game theory to balance the benefits of the government, taxi company, taxi drivers and customs;

- Using neural network to forecast the oil price;

- Using questionnaire to acquire the taxi drivers' psychological change;

- Using GIS to grasp the distribution of the taxis in the city;

- Using MAS to simulate the taxi drivers' behavior response intensity according to the fluctuations of the taxi drivers' income.

Dynamic visualized structures of the concerned topic may reinforce the stimulation and facilitate further thinking during complex problem solving process. The evolving diagrams may also help to find some hidden structures to aid qualitative scenario or hypotheses forming which is the foundational work of quantitative modeling.

\subsection{Multi-agent System for Quantitative Simulation}

Here, only MAS method in StarLogo platform developed by MIT (downloaded at http://education.mit.edu/starlogo/) is used to simulate the taxi drivers' behavior response intensity according to the fluctuations of the taxi drivers' income. The only agent (taxi drivers) is categorized into 6 groups by level of incomes.

The behaviors of each agent and the conditions of the system are defined as following.

- Initial condition: each agent owns energy to afford his daily life. Here energy of each agent is defined as its income.

- Terminate condition: after a step, here meaning one day, the energy of agent will be decreased according to a const number of energy. As energy is less than the minimum cost for a daily life, agent stops movement. 


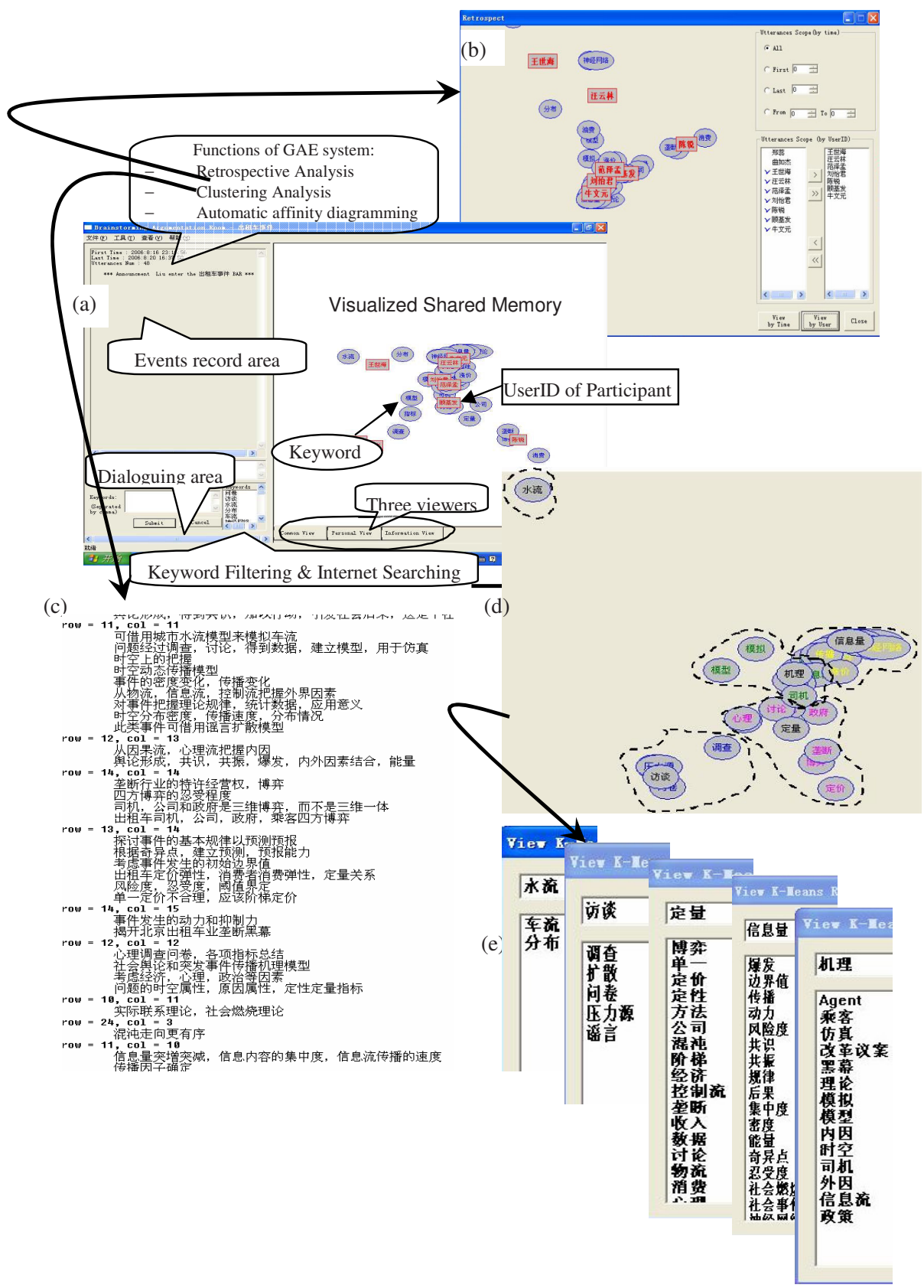

Fig. 1. Client Window of BAR. (a) Main client window. (b) Main client window. (c) Automatic affinity diagramming.(d) Clustering analysis $(K=5)$.(e) List of a cluster of keywords by (d). 
- Hypothetic condition: it is the process of modeling.

- $\quad$ assuming the initial oil price equal to 5 RMB Yuan, which shows the tendency of gradually rising;

- $\quad$ adjusting the unit price 1.6 RMB Yuan per kilometer to 2 RMB Yuan per kilometer, which causes the passengers reducing and the rate of unload driving increasing. We assumed that when the unit price is 1.6 RMB Yuan per kilometer, the rate of unload driving equals to $20 \%$, which will go up to $40 \%$ when the unit price is 2 RMB Yuan per kilometer;

- canceling the subsidy of oil price, which means the taxi drivers' income will be reduced by 670 RMB Yuan per month;

- embodying the taxi drivers' psychological change via reducing of their income. Different income level of taxi drivers was given the different degree of energy reducing.

- Simulation experiment analysis: Agents who own the lower energy (income) will stop movement in 2 days, as shown in Fig 2(a), (b), and the middle level of agents will stop in 3 days, as Fig 2(c), (d) showed, while the high energy agents last 4 days, as Fig 2(e), (f) showed. More strictly the conditions assuming, sooner the agents stop movement.

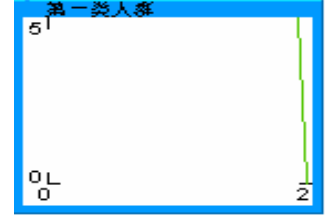

(a)

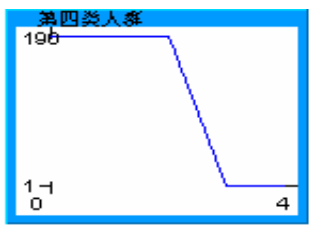

(d)

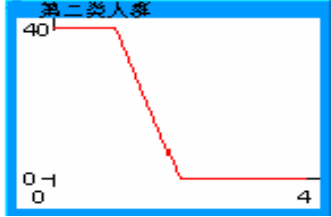

(b)

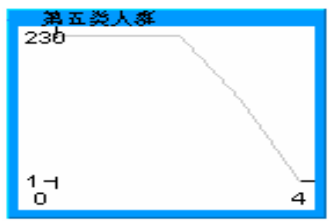

(e)

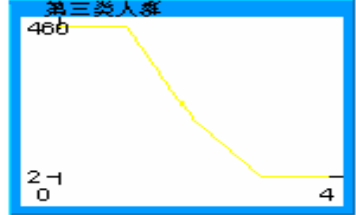

(c)

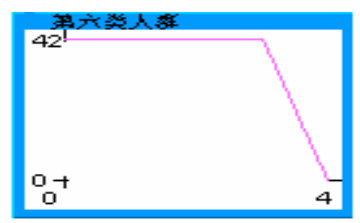

(f)

Fig. 2. Taxi Drivers' Behavior Response Intensity by MAS Simulation

Till now, the practice of meta-synthesis approach to the concerned problems is addressed. We wish the above analysis could propose an effective perspective and way for decision makers to aid them to make practical and feasible policies, such as suitable taxi price adjustments, to satisfy both the taxi companies and the taxi drivers.

\section{Concluding Remarks}

In this paper, we are concerned to explore a computational scheme and provide one kind of concept and some demonstrations for complex problem solving by meta-synthesis 
approach. Such kind of work aims to provide different perspectives for some systemic solutions toward social issues (topics about taxi driver) unlike traditional way. Qualitative scenario or hypotheses forming for those problems through GAE is useful, advanced and unique. GAE acts as a platform promoting experts to exchange ideas, stimulating their creativity and enhancing argumentation effects. Simultaneously, meta-synthetic modeling is also a core activity used in describing and solving problems based on assumptions.

Lots of further works are under exploration, such as better human-machine interaction, evolving process of keyword network to detect the pathway of the concerned issues which can be absorbing some ideas from Chance Discovery [15], and applying more modeling methods and skills to tackle with the complex or unstructured problems, etc. More experiments and models will also be undertaken for verification and validation of the capacities of these computational scheme based on the meta-synthesis approach in practice.

Acknowledgments. This work was granted financial support from China Postdoctoral Science Foundation.

\section{References}

1. Qian, X. S., Yu J. Y., Dai R. W.: A new Discipline of Science - the Study of Open Complex Giant Systems and its Methodology, Chinese Journal of Systems Engineering \& Electronics, Vol. 4, No. 2 (1993) 2-12

2. Gu, J. F., Tang,X. J.: Some Developments in the Studies of Meta-Synthesis System Approach, Journal of Systems Science and Systems Engineering, Vol.12, No.2 (2003) 171-189

3. Gu, J. F., Tang X. J.: Meta-synthesis approach to Complex System Modeling, European Journal of Operational Research, Vol.166, No.33 (2005) 597-614

4. Mase, K., Sumi, Y., Nishimoto, K.: Informal Conversation Environment for Collaborative Concept Formation. In: Ishida, T. (eds.): Community Computing: Collaboration over Global Information Networks, Wiley, New York (1998) 165-205

5. Hori, K.: A System for Aiding Creative Concept Formation. IEEE Transactions on Systems, Man and Cybernetcis, Vol.24, No. 6 (1994) 882-893

6. Nishisato S.: Analysis of categorical data: Dual scaling and its applications. University of Toronto Press, Toronto (1980) 1-53

7. Liu, Y. J., Tang, X, J.: Computerized Collaborative Support for Enhancing human's Creativity for Networked Community. Proceedings of Workshop on Internet and Network Economics, Lecture Notes in Computer Science, Springer-Verlag Berlin Heidelberg, Hong Kong, (2005) 545-553

8. Ohiwa H, Takeda N, Kawai K, Shiomi A. KJeditor: a card-handing tool for creative work support. Knowledge-Based Systems, Vol.10, (1997) 43-50

9. Tang, X. J., Liu, Y. J.: A Prototype Environment for Group Argumentation. In the Proceedings of the 3rd International Symposium on Knowledge and Systems Sciences, Shanghai (2002) 252-256

10. Duda, R. O., Hart, P. E., Stork, D. G.: Pattern Classification. Wiley, New York (2001) 526-528 
11. Tang, X. J., Liu, Y. J.: Exploring computerized support for group argumentation for idea generation. In: Nakamori, Y. et al. (eds.): Proceedings of the fifth International Symposium on Knowledge and Systems Sciences, Japan (2004) 296-302

12. Tang, X. J., Liu, Y. J.: Computerized Support for Qualitative Meta-synthesis as Perspective Development for Complex Problem Solving. In Adam, F. et al. (eds.): Creativity and Innovation in Decision Making and Decision Support (proceedings of IFIP WG 8.3 International Conference on Creativity and Innovation in Decision Making and Decision Support), Vol.1, Decision Support Press, London, (2006) 432-448

13. Ackoff,R L, Sasieni, M. W.: Fundamentals of Operations Research. John Wiley \& Sons, 1968

14. Tang, X. J., Nie, K., Liu,Y. J.: Meta-synthesis Approach to Exploring Constructing Comprehensive Transportation System in China, Journal of Systems Science and Systems Engineering, Vol.14, No.4 (2005) 476-494

15. Ohsawa, Y.: Product Designed on Scenario Maps Using Pictorial KeyGraph. WSEAS Transaction on Information Science and Application, Vol.3 No.7 (2006) 1324-1331 